\title{
A quasi-one-dimensional model for the centrifugal compressors performance simulations
}

\author{
Wenhai $\mathrm{Du}^{1}$, Yucheng $\mathrm{Li}^{1}$, Longfei $\mathrm{Li}^{1}$, Giulio Lorenzini2 ${ }^{*}$ \\ ${ }^{1}$ Beijing Institute of Petrochemical Technology, Department of Mechanical Engineering, Qingyuan North Road 19, Daxing \\ District, Beijing 102617, China \\ ${ }^{2}$ University of Parma, Department of Engineering and Architecture, Parco Area delle Scienze 181/A, Parma 43124, Italy
}

Corresponding Author Email: giulio.lorenzini@unipr.it

https://doi.org/10.18280/ijht.360202

Received: 14 December 2017

Accepted: 24 April 2018

\section{Keywords:}

centrifugal compressor, quasi-onedimensional, numerical simulation, loss models

\begin{abstract}
This paper presents a quasi-one-dimensional numerical tool to simulate the performance of the centrifugal compressors. The current model is especially useful since it could offer the reliable prediction for the centrifugal compressor performance only based on the simple geometries. An adapted version of the Euler equations solved at mid-span by a time-marching, finitevolume method, is applied in the model. The inviscid effect, the viscous effect and the geometry variation effect in the centrifugal compressor are expressed by the source terms in the Euler Equations. In the study, different loss sources in the centrifugal compressor are analyzed and estimated by empirical correlations. Two different centrifugal compressors are applied to validate the current model and the numerical simulations are compared to experimental data. The results suggest that the model provides a valuable tool for evaluating the centrifugal compressor performance during the preliminary design and optimization process.
\end{abstract}

\section{INTRODUCTION}

The centrifugal compressors have been widely applied in various domains such as aerospace, automotive industry, oil and gas industry, and power generation because of high efficiency, compact size and wide flow range. The highperformance centrifugal compressor is more appealing to the turbocharger and micro gas turbine industries due to the fact that the existing centrifugal compressor has no ability to meet the required performance target in terms of efficiency and flow range. Therefore it is necessary to develop a fast, robust and general simulation tool to offer reliable predictions for radial machines. First of all, this tool could be used to perform parametric studies to demonstrate the influences of the geometry changes on the centrifugal compressor performance both design and off-design conditions, especially during the preliminary design and optimization processes [1]. In addition, it has the ability to predict the overall performance of a combination system where centrifugal compressor is a component of such a system [2-3].

Most of the centrifugal compressor models are based upon space-marching techniques. They stack duct and bladed elements, connected through physical variables and relationships. The impeller analysis often relies on the jetwake model [4-5], but it has proved to be less accurate than the meanline computations using correlations [6]. In this paper, the current program resulting from the extension of an axial compressor and turbine model [7-8], is developed to model the stationary, transient and dynamic simulation operation of a centrifugal compressor.

The tool is able to compute the flow through a centrifugal compressor, with detail at the blade row level. It relies upon a much customized version of the Euler equations, expressed in curvilinear, still quasi-one-dimensional and conservative form. The numerical model is applied to two different centrifugal compressors and the simulation results are compared to the experimental data. The results illustrate that the model with the tuning coefficient in empirical correlations, has the capabilities to correctly capture the performance of the centrifugal compressor.

\section{MODEL EQUATIONS}

Usual quasi-one-dimensional models for axial compressors only allow reasonable deflections of the fluid, because only the axial speed is taken into account. Strong tangential speeds lead to undervalued total variables and distort the solution. A fourth equation dealing with the tangential momentum conservation was then added to the three classical quasi-1D Euler equations expressing the conservation of mass, axial momentum and total energy. The cylindrical system of equations was replaced by a curvilinear one in order to extend the application field to radial flows. The equations are discretized at mid-span and the flow is assumed to axisymmetric. Interactions between the working fluid and the centrifugal compressor are introduced into the equations by the source terms. The blade force source terms are applied to consider the energy from the blades to the working fluid, while the flow viscidity influences are represented by the friction source term. The empirical correlations provide the information to evaluate the velocity triangles at mid-span and these triangles yield the necessary information for the assessment of the source terms.

\subsection{General formulation}

The quasi-1D Euler equations are applied in the curvilinear $(\mathrm{m}, \mathrm{n}, \theta)$ coordinate system. The upper and lower boundaries 
of the control volume $\Gamma$ shown on figure 1 are solid walls. The only "porous" boundaries of $\Gamma$ are therefore the annular surfaces 1 and 2 .

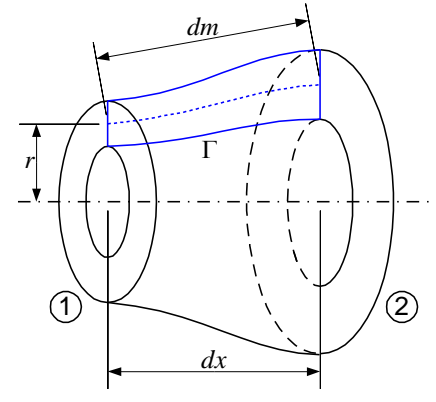

Figure 1. Elementary control volume

In vector, non-dimensional, conservative form, these equations are written as

$$
\frac{\partial \mathbf{U}}{\partial t}+\frac{\partial \mathbf{F}}{\partial m}=\mathbf{Q}
$$

$\mathbf{U}=\left[\begin{array}{c}\rho S \\ \rho V_{m} S \\ \rho V_{\theta} S \\ \rho e^{\circ} S\end{array}\right] \quad \mathbf{F}=\left[\begin{array}{c}\rho V_{m} S \\ \left(p+\rho V_{m}^{2}\right) S \\ \rho V_{m} V_{\theta} S \\ \rho h^{\circ} S\end{array}\right]$

The system of equations (1) is able to describe the evolution of the working fluid through any type of machine, axial, radial or mixed-flow if the appropriate source terms are supplied to the model. The source terms Q could be divided into three distinct contributions, categorized according to their physical meaning.

$$
Q=Q_{b}+Q_{f}+Q_{g}
$$

Namely, $Q_{b}, Q_{f}$ and $Q_{g}$ represent the blade force source term, friction force source term and geometrical source term respectively. The detailed source terms have been applied for axial compressors and turbines [8].

\subsection{Method of the velocity triangles}

The predictive capabilities of the method are highly depending on the source terms, which are brought by the estimation of the velocity triangles for the compressor blade rows at each time step. The flow variables from the current iteration and the blade geometrical data are used as inputs for the empirical correlations. The total pressure loss coefficients and the slip factor model are evaluated through open literature correlations in order to determine the aerodynamic parameters in the preliminary design process. The modular conception of the computer code allows the introduction of tuning coefficients in order to particularize the loss and slip factor models for a given hardware to produce a reliable performance prediction for the centrifugal compressor. This has already been done for axial compressors [7].

Empirical equations are established and studied by many previous researchers including Stodola [9], Cordier [10], Rodgers [11], and Wiesner [12]. Rodgers and Sapiro carried out the detailed parametric study on compressor performance and correlated the efficiency of the single stage centrifugal compressor with four major parameters: inlet specific speed, impeller tip diameter, inducer tip relative Mach number, and exit discharge Mach number [13]. For centrifugal compressor impellers, this loss coefficient includes several contributions:

$$
\begin{aligned}
& \varpi_{t o t}=\varpi_{i n c}+\varpi_{b l}+\varpi_{s f}+\varpi_{d f}+\varpi_{l k}+\varpi_{r c}+\varpi_{c l} \\
& \text { - } \quad \text { inc : incidence losses } \\
& \text { - } \quad b l \text { : losses due to the blade loading } \\
& \text { - } \quad s f \text { : skin friction losses inside the blade channel } \\
& \text { - } \quad d f \quad \text { : disc friction losses } \\
& \text { - } \quad l k \text { : leakage losses } \\
& \text { - } r c \text { : recirculation losses } \\
& \text { - } \quad c l \text { : clearance losses }
\end{aligned}
$$

The incidence loss is evaluated by the correlation proposed by Conrad, which is represented as following:

$$
\Delta \mathrm{H}_{\mathrm{inc}}=f_{\text {inc }} w_{u i}^{2} / 2
$$

where $f_{i n c}$ is the incidence factor and $w_{u i}$ is the tangential component of impeller inlet relative velocity.

The blade loading loss is developed due to the negative gradient of velocity in the boundary layer and the empirical correlation is computed by Coppage [12].

$$
\Delta \mathrm{H}_{b l}=0.05 D_{f}^{2} U_{2}^{2}
$$

The diffusion factor $D_{f}$ in the equation is similar to the one of Lieblein [14].

The skin friction loss results from the action of shearing forces on the walls of the impeller due to the turbulent fluid friction. The loss could be evaluated by the equation of Jansen.

$$
\Delta \mathrm{H}_{s f}=5.6 C_{f} \frac{L_{b}}{D_{h y d}} V_{2 m}^{2}
$$

where $C_{f}$ is the skin friction coefficient, $L_{b}$ is the impeller flow length and $D_{h y d}$ is impeller average hydraulic diameter.

The disc friction loss mechanism occurs as a result of adhesive forces between the rotating disk and the fluid in the surrounding enclosure. The disc friction enthalpy loss used is the one of Daily and Nece; it was selected by Oh et al. [15].

$\Delta h_{d f}^{o}=f_{d f} \frac{\bar{\rho} r_{2}^{2} U_{2}^{3}}{4 \dot{m}}$

With

$\bar{\rho}=\frac{\rho_{1}+\rho_{2}}{2}$

$f_{d f}=\left\{\begin{array}{l}\frac{2.67}{\operatorname{Re}_{d f}^{0.5}}, \operatorname{Re}_{d f}<3 \times 10^{5} \\ \frac{0.0622}{\operatorname{Re}_{d f}^{0.2}}, \operatorname{Re}_{d f} \geq 3 \times 10^{5}\end{array}\right.$ 
$\operatorname{Re}_{d f}=\frac{U_{2} r_{2}}{v_{2}}$

The leakage loss comes from the leakage fluid through the compressor seals to the regions of low pressure and is proposed by Aungier as following [4].

$\Delta h_{l k}^{o}=\frac{\dot{m}_{c l} U_{c l} U_{2}}{2 \dot{m}}$

With

$U_{c l}=0.816 \sqrt{\frac{2 \Delta p_{c l}}{\rho_{2}}}$

$\Delta p_{c l}=\frac{\dot{m}\left(r_{2} V_{\theta 2}-r_{1} V_{\theta 1}\right)}{Z_{b l} \bar{r} \bar{h}_{v} L_{\theta}}$

$\bar{r}=\frac{r_{1}+r_{2}}{2}$

$\bar{h}_{v}=\frac{h_{v 1}+h_{v 2}}{2}$

$\dot{m}_{c l}=\rho_{2} Z_{b l} \varepsilon L_{\theta} U_{c l}$

The recirculation loss is associated with the recirculation of air from the diffuser to the impeller and is set up by $\mathrm{Oh}$ et al. [15];

$\Delta h_{r c}^{o}=8 \times 10^{-5} \sinh \left(3.5 \alpha_{2}^{3}\right) D_{f}^{2} U_{2}^{2}$

The clearance loss is considered when the flow escapes from impelling by the clearance between the blades and the outer casing of the centrifugal compressor. It could be expressed by Rodgers as following:

$\Delta H_{c l}=0.1 \frac{s}{b_{2}} U_{2}^{2}$

The outlet tangential speed is therefore fully defined if the slip factor can be deduced from the compressor geometry. The slip factor is deduced from figure 2 and is defined as following:

$\sigma=1-\frac{V_{\text {slip }}}{U_{2}}$

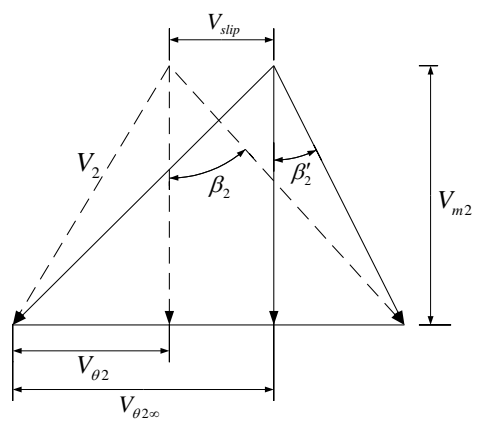

Figure 2. Impeller discharge velocity triangles
There exist a variety of slip factor correlations in the literature. A recent and remarkable article presents a unified correlation that is shown to supersede the correlations of Stodola, Stanitz, Wiesner or Busemann as following [15-16].

$$
\sigma=1-\frac{1}{1+5 \frac{\left(1-\frac{r_{1}}{r_{2}}\right) Z_{b l}}{2 \pi \sqrt{\cos \beta_{2}^{\prime}}}}
$$

In the case of vaned diffusers, the deviation angle is computed through adapted axial-flow compressor correlations, with a reference deviation at minimum-loss conditions, and then an incidence contribution [8].

\section{EQUATION SOLVER}

The calculation domain is discretized in the streamwise direction at mid-span of the blade rows. The computation domain is modified to take into account the curvilinear frame of reference. The blade rows are generally divided internally into control volumes not only in the non-bladed regions but also inside of each blade row, in order to obtain moderate flow deflections for each elementary cell. In each control volume, the integral form of the Euler equations is written and the conservative variables are expressed at the cell center. A quadratic reconstruction scheme with a hybrid limiter and a Roe's flux splitting are used to extrapolate the variables and to calculate the fluxes at the cell interfaces [17].

The real mesh counts more than three hundred mesh cells and is less readable. It was found that the large deflections imposed inside the blade zones to the working fluid had to be distributed over an important number of inner blade cells to keep the truncation errors at an acceptable level. The rest of the machine was meshed accordingly to obtain a homogeneous 1D spatial discretization.

A time-marching method allows the flow to evolve towards a stationary solution. Both explicit and implicit schemes are implemented. The explicit scheme is a fourth-order accurate Runge-Kutta. The implicit scheme is based on an Euler singlestep forward in time differentiation. For a stationary simulation, a large CFL number can be applied to speed up the calculation. The annulus boundary layer model proposed by Stratford is implemented [7]. The boundary conditions applied at the inlet and the outlet of the compressor are displayed in Table 1:

Table 1. Imposed boundary conditions

\begin{tabular}{ccc}
\hline & Inlet & Outlet \\
\hline input & $P_{\text {in }}^{0}, T_{\text {in }}^{0}, \alpha$ & $P_{\text {out }}$ \\
\hline
\end{tabular}

\section{NUMERICAL SIMULATION}

The present preliminary design tool was validated by two centrifugal compressor rigs from the open literature. The first test case comes from the Came's high speed centrifugal compressor rig [18], and the second centrifugal compressor is the CC3 impeller scaled up by McKain [19]. The centrifugal compressor of Came has high rotation speed and high pressure 
ratio, while the $\mathrm{CC} 3$ impeller is an intermediate pressure ratio. Both of the two centrifugal compressors have the subsonic inlet condition and represent the specific application in the gas turbines and turbochargers.

Came's centrifugal compressor has a rotating speed of $40000 \mathrm{RPM}$, the mass flow rate of $1.81 \mathrm{~kg} / \mathrm{s}$ and the total pressure ratio of 6.5 at the design point. The assumed stage isentropic efficiency is about $78 \%$ and the dimensionless specific speed is 0.53 . The CC3 impeller is designed to obtain the stage total pressure ratio of 4.0 at a design mass flow rate of $4.54 \mathrm{~kg} / \mathrm{s}$ and a rotation speed of 21,789 RPM. The stage isentropic efficiency is about $83 \%$ and the dimensionless specific speed is 0.6 . Both of the two centrifugal compressors are designed with backswept trailing edges and splitter blades. The Came's impeller has 17 full blades and 17 splitter blades, while the CC3 impeller has 15 full blades and 15 splitter blades. Both of two centrifugal compressors have the vane diffuser. The detailed design parameters of the two centrifugal compressors are listed in the Table 2.

Table 2. Design parameters for the two centrifugal compressors

\begin{tabular}{cccc}
\hline & Parameter & Came & CC3 \\
\hline Rotation speed & $\mathrm{N}(\mathrm{rpm})$ & 40,000 & 21,789 \\
\hline Mass flow rate & $\mathrm{m}(\mathrm{kg} / \mathrm{s})$ & 1.81 & 4.54 \\
\hline Total pressure ratio & $\mathrm{Ptr}$ & 6.5 & 4.1 \\
\hline $\begin{array}{c}\text { Isentropic } \\
\text { efficiency }\end{array}$ & $\eta$ & 0.78 & 0.83 \\
\hline $\begin{array}{c}\text { Blade number } \\
\text { full/splitter }\end{array}$ & $\mathrm{Z}_{\mathrm{f}} / \mathrm{Z}_{\mathrm{s}}$ & $17 / 17$ & $15 / 15$ \\
\hline Inlet hub radius & $\mathrm{R}_{1 \mathrm{~h}}(\mathrm{~mm})$ & 30.48 & 41.4 \\
\hline Exit blade angle & $\beta_{2 b}(\mathrm{deg})$ & 30.0 & 50.0 \\
\hline
\end{tabular}

The Came's centrifugal compressor is firstly applied to validate the numerical model. A parametric identification procedure will be conducted to demonstrate the generalization capabilities of the current tool. The objective of this procedure is to optimize the total pressure ratio and adiabatic efficiency according to the experimental results on the $100 \%$ nominal speed data. It is supposed that the optimized loss and deviation models will be able to provide a good prediction for the entire range of mass flows and rotation speeds. The loss coefficient and the slip factor coefficient are included in the optimization process in order to satisfy the experimental results.

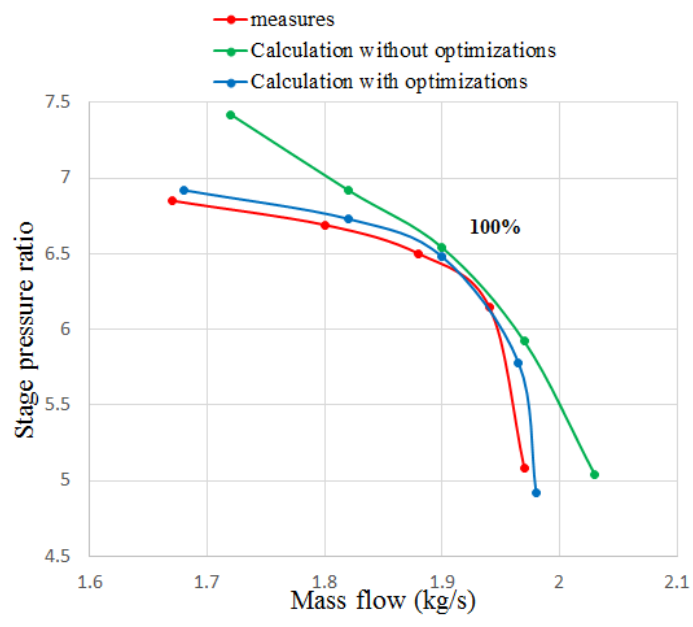

Figure 3. Comparison the total pressure ratio vs. mass flow of Came's compressor at 100\% RPM

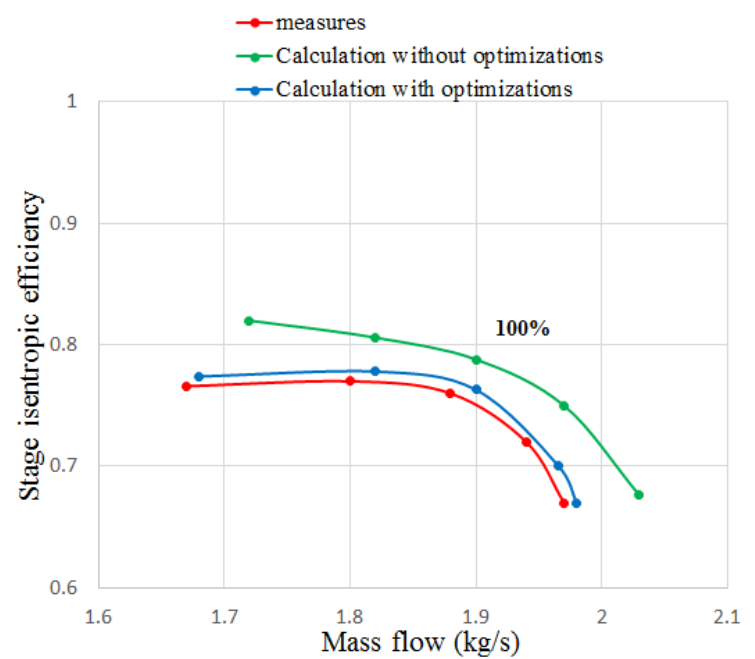

Figure 4. Comparison the isentropic efficiency vs. mass flow of Came's compressor at 100\% RPM

Figures 3 and 4 show that the total pressure ratio and isentropic efficiency with the optimized correlations are compared with the standard correlations results at $100 \%$ rotation speed. In figure 3 , it can be found that the total pressure ratio with the 'standard correlations' is overestimated at reduced mass flow and high mass flow region, which indicates that the slip factor has been overestimated. From figure 4, the adiabatic efficiency is also overestimated with the standard correlations, showing only a constant offset that can easily be compensated by tuning the recirculation loss coefficient. During the optimization process, the slip factor and pressure loss coefficients have been optimized according to the experiment results. With the optimized coefficients, the numerical results are quite satisfactory at $100 \%$ rotation speed in figures 3 and 4 .

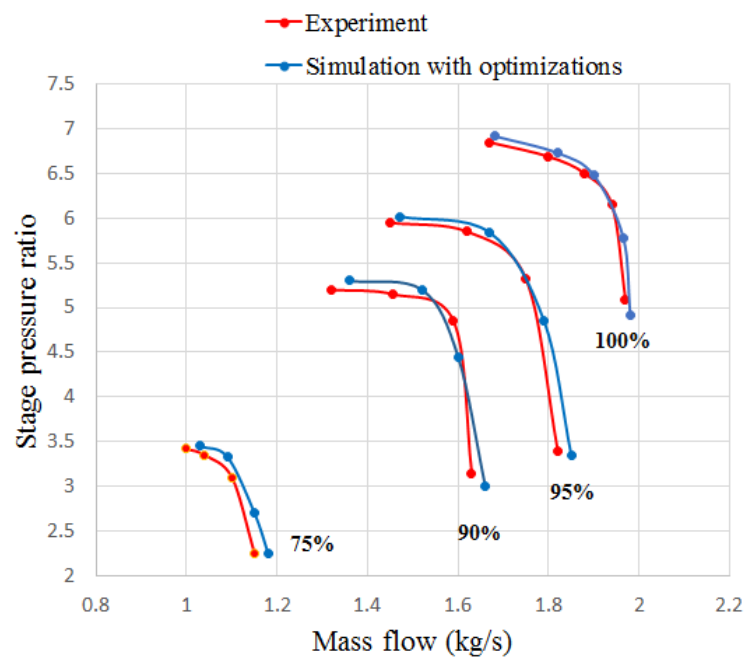

Figure 5. Total pressure ratio vs. mass flow with the optimized correlations for Came's compressor

For the Came's centrifugal compressor, the predicted performance maps with the optimized correlations show the good trends comparing to the experiment results at $75 \%, 90 \%$, $95 \%$ and $100 \%$ design rotating speed. The figures 5 and 6 show the numerical and experimental results, the total pressure ratio as well as for the isentropic efficiency. 
Finally, figures 7 and 8 present the comparison between the measured results and numerical simulations for the total pressure ratio and adiabatic efficiency respectively for the CC3 centrifugal compressor. Although CC3 centrifugal compressor has an intermediate pressure ratio, it can be found that the similar numerical predictions are obtained with the optimized correlations, which demonstrates the generalities of the current tool.

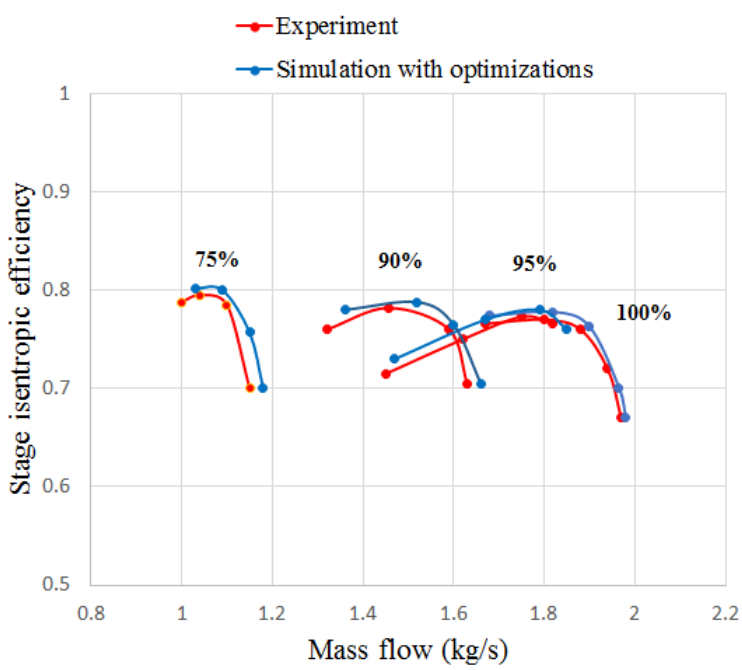

Figure 6. Isentropic efficiency vs. mass flow with the optimized correlations for Came's compressor

\section{CONCLUSIONS}

A quasi-one dimensional model for centrifugal compressor has been presented. The simulation tool is developed from the extension of the previous axial compressor, thus the equations have been transposed from cylindrical to curvilinear coordinates for the centrifugal compressor in order to enhance the simulation accuracy. The current tool uses only the machine geometry and the functional parameters such as the rotation speed, the inlet total pressure and temperature, or the outlet static pressure, to simulate the flow taking place inside the machine flowpath.

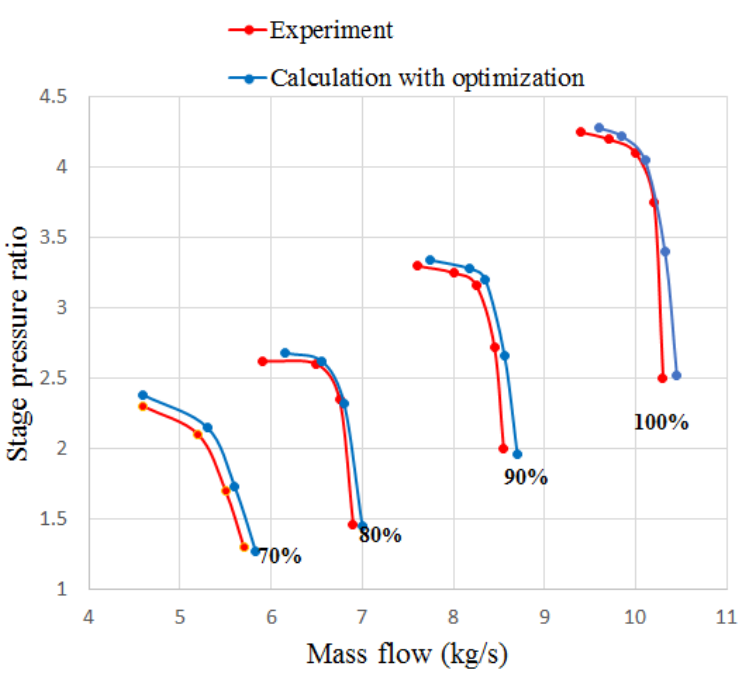

Figure 7. Total pressure ratio vs. mass flow with the optimized correlations for CC3 compressor

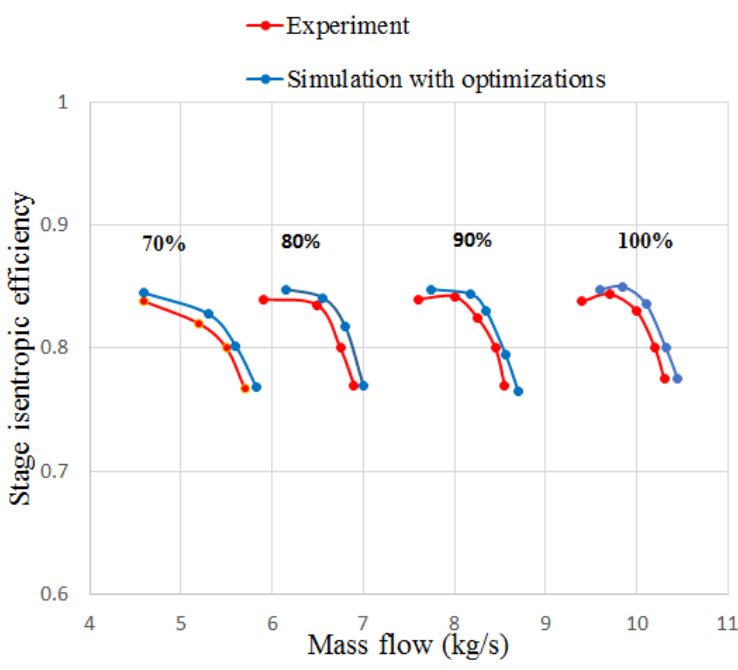

Figure 8. Isentropic efficiency vs. mass flow with the optimized correlations for $\mathrm{CC} 3$ compressor

The total pressure loss and slip factor correlations described in the paper cover a large part of the physical phenomena occurring during the stationary operation of a centrifugal compressor. Meanwhile, the open literature correlations introducing empirical information into the simulation can be easily exchanged for a manufacturer's own correlations, or tuned by means of coefficients to be optimized. The Came's centrifugal compressor and CC3 centrifugal compressor are selected as the validation test case. The total pressure loss and slip factor coefficient are optimized according to the experiment performances at 100\% RPM of Came's centrifugal compressor, the predicted performances of the two centrifugal compressors during the entire rotation speeds are quite satisfying. It indicates that the current code is useful during the preliminary design process of the centrifugal compressor.

\section{ACKNOWLEDGMENT}

This research has been supported by Beijing Natural Science Foundation (3182009).

\section{REFERENCES}

[1] Whitfield A, Baines NC. (1990). Design of radial turbomachines. Longman Scientific \& Technical.

[2] Du W, Léonard O. (2011). A quasi-one-dimensional CFD model for multistage compressors. ASME 2011 Turbo Expo: Turbine Technical Conference and Exposition 17: 7-20. https://doi.org/10.1115/gt201145497

[3] Wenhai DU, Zhu JQ, Léonard O. (2012). Dynamic simulations of post-stall performance in multistage axial compressors. Journal of Thermal Science 21(4): 311321. https://doi.org/10.1007/s11630-012-0549-y

[4] Aungier RH. (2000). Centrifugal compressors : a strategy for aerodynamic design and analysis. ASME Press.

[5] Djedai H, Mdouki R, Mansouri Z, Aouissi M. (2017). Numerical investigation of three-dimensional separation control in an axial compressor cascade. International Journal of Heat \& Technology 35(3): 657-662. https://doi.org/10.18280/ijht.350325 
[6] Frigne P, Van DBR. (1978). One dimensional design of centrifugal compressors taking into account flow separation in the impeller. VKI Technical Note 129, pp. 28-42.

[7] Adam O, Léonard O. (2005). A Quasi-one-dimensional model for axial compressors. 17th ISABE Conference, Münich, Germany.

[8] Léonard O, Adam O. (2008). A quasi-one-dimensional cfd model for multistage turbomachines. Journal of Thermal Science 17(1): 7-20. https://doi.org/10.1007/s11630-008-0007-z

[9] Lou F, Fabian JC, Key NL. (2018). A new approach for centrifugal impeller preliminary design for aero-thermal analysis. Journal of Turbomachinery 140: 1-10. https://doi.org/10.1115/1.4038876

[10] Cordier O. (1955). Similarity considerations in turbomachines. Verlag. Dusseldorf, Germany. VDI Reports 3. https://doi.org/10.1115/cec1955-0104

[11] Rodgers C. (1964). Typical performance characteristics of gas turbine radial compressors. Journal of Engineering for Gas Turbines \& Power 86(2): 161. https://doi.org/10.1115/1.3677568

[12] Tian Y, Hu A. (2018). Study on critical speed of rotation in the multistage high speed centrifugal pumps rotors. International Journal of Heat \& Technology 36(1): 3139. https://doi.org/10.18280/ijht.360105

[13] Velásquez EIG. (2017). Determination of a suitable set of loss models for centrifugal compressor performance prediction. Chinese Journal of Aeronautics 30(5): 16441650. https://doi.org/10.1016/j.cja.2017.08.002

[14] Lieblein S. (1965). experimental flow in twodimensional cascades. NASA, SP-36 183-226. https://doi.org/10.1115/1.801926.ch5

[15] Oh HW, Yoon ES, Chung MK. (1997). An optimum set of loss models for performance prediction of centrifugal compressors. Proceedings of the Institution of Mechanical Engineers Part A Journal of Power \& Energy 211(4): $331-338$ https://doi.org/10.1243/0957650971537231

[16] Wiesner FJ. (1967). A review of slip factors for centrifugal impellers. Journal of Engineering for Gas Turbines \& Power 89(4): 558-566. https://doi.org/10.1115/1.3616734

[17] Hirsch Ch. (1988). Numerical computation of internal and external flows. Fundamentals of Numerical Discretization, John Wiley \& Sons, Chichester, UK.

[18] Came PM. (1978). Development, application and experimental evaluation of a design procedure for centrifugal compressors. Proceedings of the Institution of Mechanical Engineers 192(1): 49-67. https://doi.org/10.1243/pime_proc_1978_192_051_02

[19] Mckain TF, Holbrook GJ. (1997). Coordinates for a high performance $4: 1$ pressure ratio centrifugal compressor. NASA Contract Report 204134 https://www.researchgate.net/publication/24321070_Co
ordinates_for_a_High_Performance_41_Pressure_Ratio _Centrifugal_Compressor

\section{NOMENCLATURE}

$\begin{array}{ll}\mathrm{b}_{2} & \text { Outlet blade height }[\mathrm{m}] \\ e & \text { specific internal energy [KJ/Kg] } \\ D_{f} & \text { dimensionless diffusion factor } \\ \mathbf{F} & \text { conservative flux vector } \\ h & \text { specific enthalpy }[\mathrm{KJ} / \mathrm{Kg}] \\ L & \text { length [m] } \\ p & \text { static pressure [Pa] } \\ \mathbf{Q} & \text { source term vector } \\ q_{m} & \text { mass flow [Kg/s] } \\ r & \text { radius [m] } \\ S & \text { dimensionless solidity } \\ S & \text { flowpath cross-sectional area }\left[\mathrm{m}^{2}\right] \\ t & \text { time [s] } \\ \mathbf{U} & \text { conservative variable vector } \\ U & \text { tangential rotation speed }[\mathrm{m} / \mathrm{s}] \\ V & \text { absolute velocity }[\mathrm{m} / \mathrm{s}] \\ V o l & \text { blade row volume }\left[\mathrm{m}^{3}\right] \\ W & \text { relative velocity }[\mathrm{m} / \mathrm{s}] \\ Z_{b l} & \text { blade number }\end{array}$

\section{Greek symbols}

$\begin{array}{ll}\alpha & \text { absolute flow angle }[\mathrm{deg}] \\ \beta & \text { relative flow angle }[\mathrm{deg}] \\ \bar{w} & \text { pressure loss coefficient } \\ \rho & \text { density }\left[\mathrm{kg} / \mathrm{m}^{3}\right] \\ \sigma & \text { slip factor } \\ \varepsilon & \text { blade tip clearance }[\mathrm{m}] \\ v & \text { Kinematic viscosity coefficient }\left[\mathrm{m}^{2} / \mathrm{s}\right]\end{array}$

\section{Subscripts}

$\begin{array}{ll}\begin{array}{l}m \\ x\end{array} & \begin{array}{l}\text { meridional } \\ n\end{array} \\ r & \begin{array}{l}\text { normal } \\ \theta\end{array} \\ t & \begin{array}{l}\text { radial } \\ t\end{array} \\ 1 & \text { throat condition } \\ 2 & \text { blade leading edge } \\ & \text { blade trailing edge }\end{array}$

\section{Superscripts}

$\circ \quad$ total condition 